\title{
DATA PROCESSING FOR THE SPACE-BASED DESIS HYPERSPECTRAL SENSOR
}

\author{
E. Carmona ${ }^{\text {a, }}{ }^{,}$, J. Avbelj $^{\text {a }}$, K. Alonso ${ }^{\text {a }}$, M. Bachmann ${ }^{\text {a }}$, D. Cerra ${ }^{\text {a }}$, A. Eckardt ${ }^{\text {, }}$, B. Gerasch ${ }^{\text {a }}$ L. Graham ${ }^{\text {c }}$, B. Günther ${ }^{\text {b }}$, U. Heiden ${ }^{\text {a }}$, \\ G. Kerr ${ }^{\mathrm{a}}$, U. Knodt ${ }^{\mathrm{d}}$, D. Krutz ${ }^{\mathrm{b}}$, H. Krawcyk ${ }^{\mathrm{a}}$, A. Makarau ${ }^{\mathrm{a}}$, R. Miller ${ }^{\mathrm{e}}$, R. Müller ${ }^{\mathrm{a}}$, R. Perkins ${ }^{\mathrm{e}}$, I. Walter ${ }^{\mathrm{b}}$ \\ ${ }^{\text {a }}$ German Aerospace Center (DLR), Earth Observation Center (EOC), D-82234 Wessling, Germany - Rupert.Mueller@dlr.de \\ ${ }^{\mathrm{b}}$ German Aerospace Center (DLR), Optical Sensor Systems, Rutherfordstraße 2, 12489 Berlin, Germany - david.krutz@dlr.de \\ ${ }^{c}$ GeoCue LLC, 9668 Madison Blvd., Suite 202, Madison, AL 35758, USA - lgraham@geocue.com \\ ${ }^{\mathrm{d}}$ German Aerospace Center (DLR), Strategie und Vernetzungen, Linder Höhe, 51147 Köln, Germany - uwe.knodt@dlr.de \\ e Teledyne Brown Engineering, 300 Sparkman Drive NW Huntsville, AL 35805, USA - ray.perkins@Teledyne.com
}

\section{Commission I, WG I/4}

KEY WORDS: data processing, hyperspectral, ISS, geometric correction, atmospheric correction, rolling shutter

\begin{abstract}
:
The German Aerospace Center (DLR) and Teledyne Brown Engineering (TBE) have established a collaboration to develop and operate a new space-based hyperspectral sensor, the DLR Earth Sensing Imaging Spectrometer (DESIS). DESIS will provide spacebased hyperspectral data in the VNIR with high spectral resolution and near-global coverage. While TBE provides the platform and infrastructure for operation of the DESIS instrument on the International Space Station, DLR is responsible for providing the instrument and the processing software. The DESIS instrument is equipped with novel characteristics for an imaging spectrometer such high spectral resolution $(2.55 \mathrm{~nm})$, a mirror pointing unit or a CMOS sensor operated in rolling shutter mode. We present here an overview of the DESIS instrument and its processing chain, emphasizing the effect of the novel characteristics of DESIS in the data processing and final data products. Furthermore, we analyse in more detail the effect of the rolling shutter on the DESIS data and possible mitigation/correction strategies.
\end{abstract}

\section{INTRODUCTION}

The benefit of spaceborne imaging spectrometers has been long exploited in applications requiring precise identification of materials or aiming for a better understanding of different environmental processes. Although the high potential of this type of data, nowadays the availability of spaceborne imaging spectrometer data is limited (Guanter 2015). The situation may start to change in early 2018 when the DESIS hyperspectral instrument starts its operation in the Multi-User System for Earth Sensing (MUSES) platform on board of the ISS.

The DESIS instrument is the result of a collaboration between the German Aerospace Center (DLR) and Teledyne Brown Engineering (TBE) in USA. Based on this agreement, DLR is responsible for providing the DESIS instrument and TBE will be responsible for its installation and operation on the MUSES platform. DESIS data will be commercially available to customers of TBE and at the same time TBE will license the instrument data to DLR for scientific and humanitarian use.

DLR will also provide the processing software that will be used in the two data centers that will be established for processing and archiving DESIS data. One is managed by TBE and located in Huntsville, Alabama, and one is managed by DLR and located in Neustrelitz, Germany. In close collaboration, TBE and DLR will share calibration data and will provide consistent data products to their respective users.

\section{THE MUSES PLATFORM}

MUSES is a precision Earth-pointing platform for remote sensing developed by TBE as a commercial venture for the ISS (Perkins R., 2016). The MUSES platform provides accommodation for up to four instruments in four containers, two for large payloads and two for small payloads (Figure 1). The platform is attached at the EXPRESS Logistics Carriers (ELC-4) starboard of the ISS, and provides attitude and position sensing, data downlink, and other core services for each payload. MUSES is equipped with two gimbals that allow the platform to rotate around two perpendicular axes, allowing for $\pm 25^{\circ}$ forward / backward view in one axis and $45^{\circ}$ backboard (port) view and $5^{\circ}$ starboard view in the other axis.

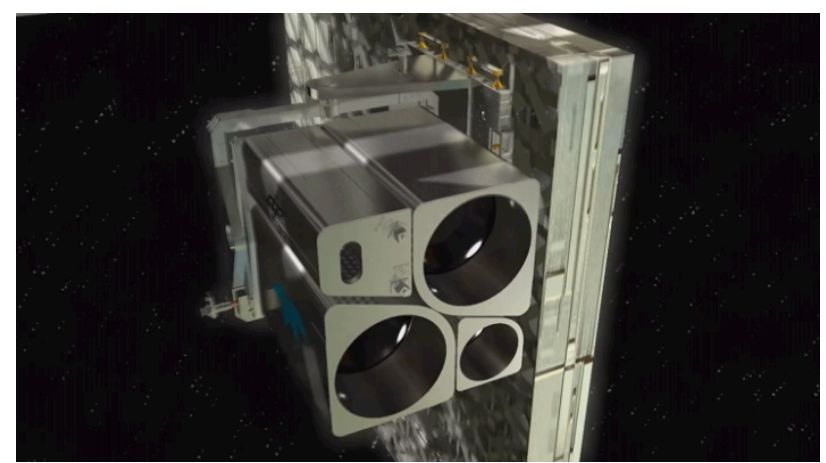

Figure 1. View of the MUSES platform equipped with four instruments (C) Teledyne Brown Engineering. All rights reserved.)

\footnotetext{
* Corresponding author
} 
MUSES will provide a pointing accuracy below 30 arc seconds (about $60 \mathrm{~m}$ on ground for $400 \mathrm{~km}$ altitude) and a timing precision of $\pm 250 \mu \mathrm{s}$ with the ISS GPS. The viewing capabilities of MUSES allow scanning about $90 \%$ of the populated Earth with a 3-5 days average cadence. MUSES launch is scheduled for April 2017.

\section{THE DESIS INSTRUMENT}

The DESIS instrument is realized as a pushbroom imaging spectrometer sensitive in the Visible to Near Infrared (VNIR) spectral range (from 400 to $1000 \mathrm{~nm}$ ). It can reach a spectral sampling distance of $2.55 \mathrm{~nm}$ employing a 2-dimensional back illuminated Complementary Metal Oxide Semiconductor (CMOS) detector array from BAE Systems (CIS2001). Larger spectral sampling distances are configurable by means of an onboard binning of up to four spectral bands (Müller, 2016). A view of the DESIS instrument design is shown in Figure 2.

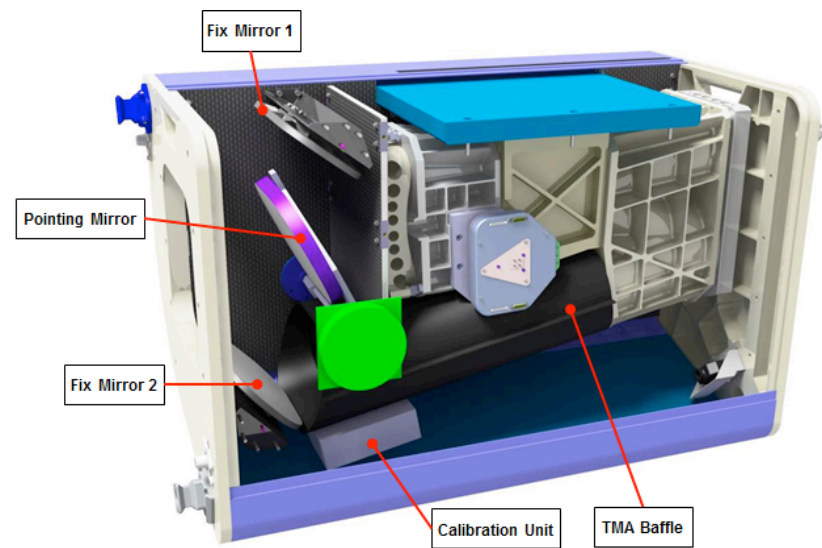

Figure 2. Design of the DESIS Instrument (C DLR-OS)

The optical design of the Instrument is based on the Offner-type grating spectrometer, widely used in hyperspectral imaging. The telescope is designed as a Three Mirror Anastigmat (TMA) with $320 \mathrm{~mm}$ effective focal length. The Ground Sampling Distance (GSD) at nadir is about $30 \mathrm{~m}$ for an ISS flying altitude of 400 $\mathrm{km}$ (reference value), resulting in a swath of about $30 \mathrm{~km}(1024$ spatial pixels in the across-track direction). The shutter mechanism is electronically realized as a Rolling Shutter (RS) what translates into different start and stop integration times for each band while keeping the same total integration time among bands. A key advantage of the RS mode is that it allows higher frame acquisition rates than the Global Shutter (GS) mode. Section 5 provides a detailed description of the DESIS RS mode and its consequences for data processing and data analysis in DESIS.

Table 1 summarizes the main design parameters of the DESIS Instrument.

\begin{tabular}{|l|l|}
\hline \multicolumn{2}{|c|}{ DESIS Instrument Design Parameters } \\
\hline $\begin{array}{l}\text { Telescope } \\
\text { F\# / Focal Length }\end{array}$ & $3 / 320 \mathrm{~mm}$, telecentric \\
\hline FOV & $4.4^{\circ}$ \\
\hline IFOV & $0.004^{\circ}$ \\
\hline GSD@ Nadir & $30 \mathrm{~m} \mathrm{@} \mathrm{400} \mathrm{km} \mathrm{(ref.} \mathrm{flight} \mathrm{altitude)}$ \\
\hline Swath@ Nadir & $\sim 30 \mathrm{~km} \mathrm{@} \mathrm{400} \mathrm{km} \mathrm{(ref.} \mathrm{flight} \mathrm{altitude)}$ \\
\hline Spectral Range & $400 \mathrm{~nm}-1000 \mathrm{~nm}$ \\
\hline
\end{tabular}

\begin{tabular}{|l|l|}
\hline $\begin{array}{l}\text { Spectral Sampling } \\
\text { and number of } \\
\text { Channels }\end{array}$ & $\begin{array}{l}2.55 \mathrm{~nm} \text { and } 235 \text { bands (no binning) } \\
5.10 \mathrm{~nm} \text { and } 117 \text { bands }(2 \times \text { binning) } \\
7.65 \mathrm{~nm} \text { and } 78 \text { bands }(3 \times \text { binning) } \\
10.21 \mathrm{~nm} \text { and } 58 \text { bands }(4 \times \text { binning) }\end{array}$ \\
\hline SNR @550nm & $\begin{array}{l}205 \text { sampled for } 2.55 \mathrm{~nm} \text { (no binning) } \\
406 \text { binned for } 10.21 \mathrm{~nm}(4 \times \\
\text { binning) }\end{array}$ \\
\hline Spatial Pixels & 1024 \\
\hline $\begin{array}{l}\text { Radiometric } \\
\text { Linearity }\end{array}$ & $>95 \%(10 \%-90 \%$ FWC) \\
\hline $\begin{array}{l}\text { MTF @ Nyquist } \\
\text { (no smearing) }\end{array}$ & $<3 \mathrm{~nm}$ \\
\hline Off-nadir capability & $\pm 15^{\circ}$ along track by POI with $1^{\circ}$ steps \\
\hline Pixel size & $24 \mu \mathrm{m}$ x $24 \mu \mathrm{m}$ \\
\hline Max. Frame rate & $232 \mathrm{~Hz}$ \\
\hline Pixel Quantization & 12 bit +1 bit for low/high gain setting \\
\hline Pointing Unit & $\begin{array}{l}\text { BRDF mode: } 11 \text { measurement } \\
\text { positions } \pm 15^{\circ}\left(\text { every } 3^{\circ}\right) \text { with } 20 \text { arc } \\
\text { min accuracy } \\
\text { FMC mode: Rotation speed } 0.6^{\circ} / \mathrm{sec} \\
\text { and } 1.5^{\circ} / \mathrm{sec} \text { with } 0.06^{\circ} \text { accuracy }\end{array}$ \\
\hline
\end{tabular}

Table 1. Design parameters of the DESIS Instrument

DESIS is equipped with a so-called POInting unit (POI) consisting of a rotating mirror in front of the entrance slit. The POI allows to change the DESIS viewing angle in the forwardbackward direction between $-15^{\circ}$ and $+15^{\circ}$, in $3^{\circ}$ steps, with respect to the nominal (nadir) view when operated in static mode (fixed mirror position). This mode will allow to take Bidirectional Reflectance Distribution Function (BRDF) products, which provide different viewing angles of a given target. The POI can also be operated in dynamic mode (moving mirror) with a viewing angle change of up to $1.5^{\circ} /$ second allowing the Forward Motion Compensation (FMC) mode where the same target on ground is continuously observed. Figure 3 shows the operation of the POI and the fixed mirrors.
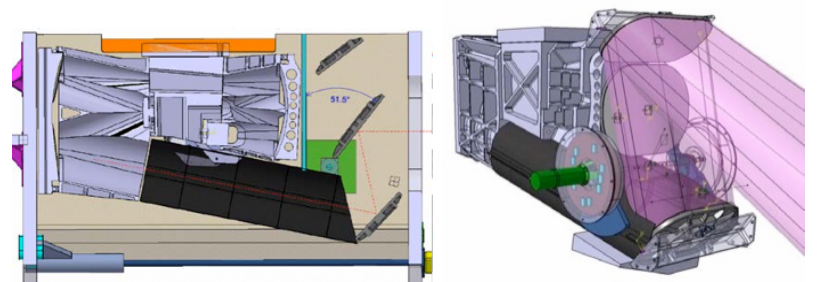

Figure 3. Operation of the POI and fixed mirrors (C DLR-OS)

The DESIS instrument is also equipped with an on-board calibration unit comprising different monochromatic and white LEDs in the 400-1000 $\mathrm{nm}$ spectral range. The calibration unit location allows the illumination of the full spectrometer FOV using the different LEDs when the POI is in calibration position. The pre-flight instrument characterization in laboratory and regular on-board measurements with the calibration unit will serve to maintain the instrument calibration during its operational lifetime.

DESIS will be launched in early 2018 and it will be robotically installed in one of the large containers of MUSES. Once installed, it is expected to start operations after a commissioning phase of 3 to 4 months. 


\section{DESIS DATA PROCESSING}

DESIS data products are defined as tiles of $1024 \times 1024$ pixels $\left(\sim 30 \times 30 \mathrm{~km}^{2}\right)$ obtained from the tiling of data take strips acquired over the Earth surface (Müller, 2016). Each of these tiles contains in turn a number of bands defined by the binning configuration of the data take. All products are generated by the same processing chain implemented at the DLR and TBE Ground Segments (GSs). As mentioned, TBE GS will be located in Huntsville (Alabama), but the data processing will be performed in a cloud-based system running on the Amazon Web Services (AWS) Cloud. DLR GS is established in different DLR locations: Neustrelitz (data processing), Berlin (calibration/monitoring) and Oberpfaffenhofen (processors). Both GSs will deliver identical products from the same original DESIS data.

Figure 4 summarizes the DESIS processing chain and associated products. These products are described in section 4.1. The novelties in the data processing resulting from the characteristics of the DESIS sensors are presented in sections 4.2 to 4.5 for the different processing steps. The effect of RS is discussed separately in section 5 .

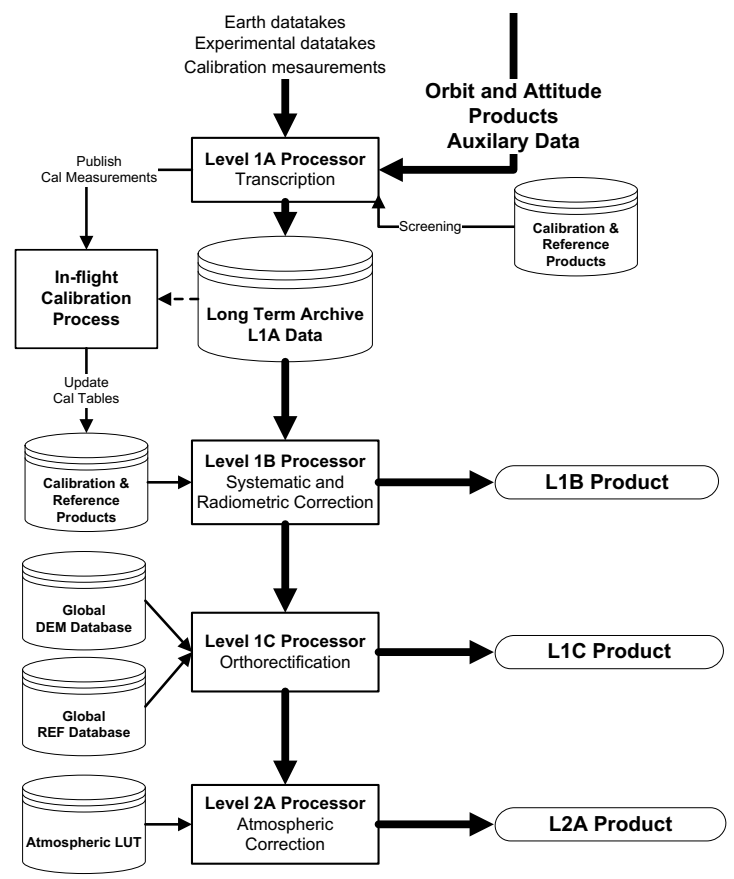

Figure 4. DESIS automatic processing chain and associated products

\subsection{DESIS Products}

The DESIS products follow the definitions of the European Space Agency (ESA) and are specified in Table 2.

\section{Product Level 0 (L0 / raw) - internal product}

Raw data after restoration of the chronological data sequence for the instrument(s) operating in observation mode, at full space/time resolution with all supplementary information to be used in subsequent processing (e.g. orbital data, health, time conversion, etc.) appended. Level 0 data are timetagged. The precision and accuracy of the time-tag shall be such that the measurement data may be localized to accuracy compatible with the user's requirements.
Product Level 1A (L1A) - internal archived product Level 0 data with corresponding radiometric, spectral and geometric (i.e. Earth location) correction and calibration computed and appended, but not applied.

\section{Product Level 1B (L1B) - deliverable product}

Level 1A data not re-sampled, quality-controlled and radiometrically calibrated, spectrally characterised, geometrically characterised, annotated with satellite position and pointing, landmarks and preliminary pixel classification (e.g. land/water/cloud mask). The Level 1B product consists of Top of Atmosphere (TOA) radiance (W.m-2.sr-1. $\mu$ m-1).

\section{Product Level 1C (L1C) - deliverable product}

Level 1B data orthorectified, re-sampled to a specified grid. Image re-sampling shall be performed using a selectable resampling method including at least bicubic convolution interpolation and nearest neighbour.

Product Level 2A (L2A) - deliverable product

Earth located pixel values converted to ground surface reflectance, i.e. after atmospheric corrections.

\section{Table 2. Definition of DESIS products}

L1A products are long-term archived in both GSs. In addition to Earth imaging scenes, L1A products also include on-board calibration measurements, Dark Current (DC) measurements and experimental products (e.g. FMC products, BRDF products) that are not generally deliverable to users. L1B, L1C, L2A products represent increasingly processing steps of the DESIS products and are only generated on demand and later delivered to the user that requested them.

Concerning data policy, L0 data are only received by TBE that has the exclusive right to license or transfer image data for commercial use. DLR has the right to task the DESIS instrument or access the TBE catalogue for humanitarian and scientific use only. The data received by DLR will be process and archived by DLR in its facilities. DLR can share DESIS scientific data with other scientific organizations exclusively for scientific purposes, being prohibited any commercial use of these data without Teledyne's prior written permission. End users of the instrument data provided to DLR for scientific use will be required to enter into the data license agreement among DLR and Teledyne, acknowledging the limited use license.

\subsection{L1A processor: Transcription processor}

The L1A Processor is responsible for combining the raw hyperspectral data with other data streams (orbit and attitude data), for extracting and interpreting those data and also for deriving additional information for long term storage of the product. L1A processor also performs the tiling of the data take strips acquired by DESIS (including the separation of DC observations if present in the data take) and the screening of status information included in the DESIS data (temperatures, voltages, etc.).

Four types of L1A products can be obtained from the raw data by the L1A processor: Earth products that can undergo further processing and are user-deliverable, Calibration products that are used for monitoring and calibration of the Instrument (not user-deliverable), DC products (not user-deliverable) and Experimental products which are Earth scenes taken in nonnominal observation modes. The experimental products are in 
general not processed to higher level products by the automatic processing chain (except for the BRDF mode).

L1A processor also generates quicklook images of the Earth products.

\subsection{L1B processor: Systematic and Radiometric Conversion processor}

This processor is responsible for correcting the data for systematic effects and converting the measured values into physical at-sensor radiance (or TOA radiance values). To that end, the processor makes use of calibration tables (pre-launch and/or from on-board calibration measurements). The steps included in this processor are the following:

- Dead pixel flagging

- Dark signal correction (obtained from dark signal measurements)

- Non-linearity response correction

- $\quad$ Spectral referencing (using spectral calibration values)

- $\quad$ Radiometric referencing

- Generation of data quality indicators

- Radiometric conversion to at-sensor radiance

- RS, smile and keystone correction

The output of this processor is a TOA radiance data cube including quality layers and metadata. A detailed explanation of the RS effect and correction can be found in section 5 .

\subsection{L1C processor: Orthorectification processor}

The L1C processor performs the so-called orthorectification process to generate map conform products, removing geometric distortions caused by the internal geometry of the sensor, the terrain being imaged and DESIS motion. The input data for the L1C processor is a L1B DESIS product. The orthoimages are generated employing the rigorous model of Direct Georeferenceing (DG) (Müller 2012). This method uses the sensor internal geometry (extensively characterized in laboratory), mounting angles, POI angle, orbit and attitude measurements from the MUSES platform and GPS information. An iterative process computes the intersection points of the sensor pixels Line-of-Sight $(\mathrm{LoS})$ and the Digital Elevation Model (DEM). This creates a grid of 3D points that are transformed to a user-selectable map projection system (e.g. UTM).

An improvement of the sensor model is achieved by using Ground Control Points (GCPs) automatically extracted from reference images of high geometric quality. Currently the Landsat- 8 panchromatic images with an absolute geometric accuracy of $12 \mathrm{~m}$ circular error at $90 \%$ confidence level and $14 \mathrm{~m}$ ground resolution is foreseen (USGS 2016) as a global reference image database for DESIS L1C processor.

The output product of this processor is an orthorectified image resampled with a user selectable resampling method $(30 \mathrm{~m}$ pixel spacing for UTM, 1 arcsec for geographic projection) to the selected map projection system. The L1C product also includes quality layers and metadata information.

\subsection{L2A processor: Atmospheric Correction processor}

The L2A processor performs the atmospheric correction of the DESIS data employing the code of ATCOR (Richter 1996, Richter 2011). L2A processor is applied on a L1C DESIS input product and uses MODTRAN-5.3.3 to model properties of the solar reflective spectrum in the $400 \mathrm{~nm}$ to $2500 \mathrm{~nm}$ range. The code includes a rigorous treatment of the coupled scattering and absorption processes. The L2A processor also offers representative aerosol models to be used during the correction (rural, urban, maritime and desert). Internally, the processor uses a MODTRAN generated database of atmospheric correction look-up tables (LUTs) with a spectral resolution of $0.4 \mathrm{~nm}$, to enable the processing of the $2.55 \mathrm{~nm}$ (no binning) band widths of DESIS.

The DESIS atmospheric correction accounts for flat and rugged terrain and includes haze/cirrus detection algorithms. The output product will be a ground reflectance data cube, maps of aerosol optical thickness and atmospheric water vapour and a set of image masks indicating: water, land, haze, cloud, shadow and snow (Makarau 2014a, Makarau 2014b).

\section{ROLLING SHUTTER EFFECT}

One particular characteristic of the DESIS instrument is the use of the RS mode for scene acquisition. RS is long used in CMOS sensors since it typically allows for faster frame rates and lower readout noise. However, it also creates the effect that different rows (different bands in DESIS) of the exposed frame are read not synchronously, resulting in different exposures.

Although the effect of RS in images and video has been long studied in conventional imaging or video cameras, this effect has not been studied yet in the context of imaging spectrometers. The question of the best correction strategy or the most cost-efficient one that could be used in DESIS remains still open. The following sections present what is the effect of the RS in the DESIS products, the baseline correction strategy presently adopted and the results obtained with this correction in the context of spectral unmixing using simulations of the DESIS data.

\subsection{RS Effect on DESIS Data}

Like in other pushbroom instruments, the 2-dimensional CMOS sensor measures simultaneously the spectra of a sampled imageline. The CIS2001 sensor employs the across direction (1024 columns) for spatial resolution of the sampled line and the along direction (235 rows) for the spectral resolution. In the case of DESIS this translates into a frame which contains a ground line (a frame) of $\sim 30 \mathrm{~km}$ (across-track) $\times 30 \mathrm{~m}$ (along-track) in all 235 spectral bands. Although the readout of the CIS2001 sensor can be performed either in GS or RS mode, only the RS mode allows the high frame rates required for DESIS operation. RS is hence the default operation mode of DESIS. The sensor is logically divided into two halves which are read starting simultaneously from the top and bottom edges towards the center of the sensor (see Figure 5).

The RS mode introduces a shift at the exposure start time of each sensor row (each spectral band) with respect to its neighbours. These shifts and the movement of DESIS on the ISS cause that the ground area captured by each band is displaced with respect to other bands. The result is that the data cube obtained from a DESIS acquisition is not aligned to a common spatial grid on ground. In order to provide an image where the 235 spectral bands (no-binning case) correspond to the same ground pixel, DESIS processing must include a step where all bands are resampled to the same spatial grid. This step is called here $R S$ Correction (RSC) and it is described in more detail in section 5.3. 

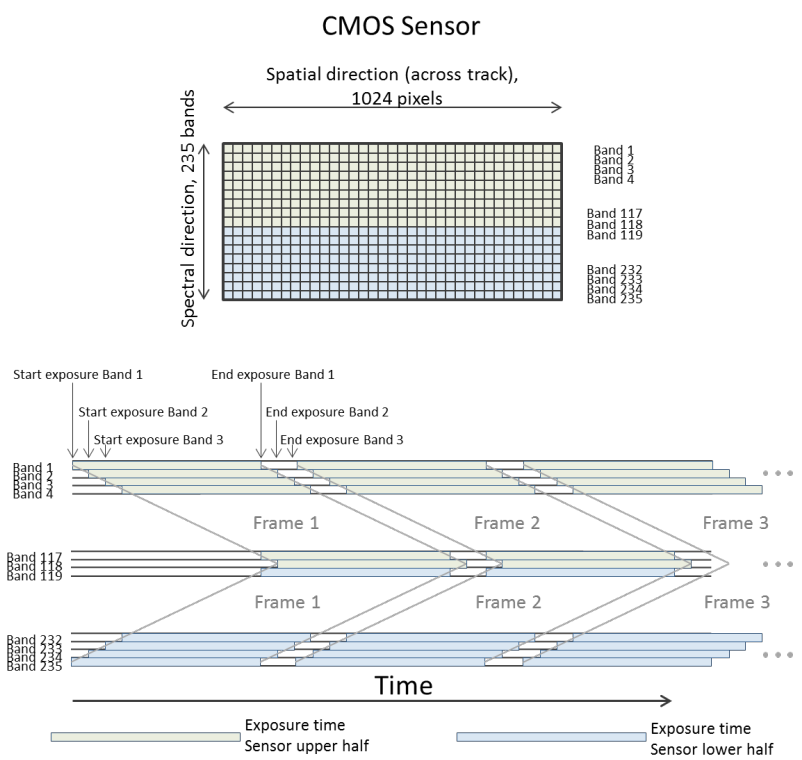

Figure 5. Schematic view of the DESIS CMOS sensor readout in RS mode

\subsection{Simulations of the DESIS instrument}

In order to study the effect of the RS and other characteristics of the DESIS instrument we have simulated DESIS data using data from another hyperspectral instrument. The HySpex VNIR1600 sensor is an airborne instrument with a spectral range and spectral resolution similar to DESIS, but much higher spatial resolution. The HySpex data are used to reproduce the parameters of the DESIS instrument (Table 1) including also the RS effect. The parameters of the HySpex VNIR sensor are summarized in Table 3.

Given the large area covered by DESIS, in comparison to HySpex, several orthorectified HySpex strips were used to compose a small DESIS scene. A gaussian smoothing according to DESIS Point Spread Function (PSF) specifications is also applied to the higher spatial resolution data from HySpex. The DESIS bands not covered by HySpex data are filled with data from the closest HySpex bands. A subset of the DESIS simulated scene is used for the studies performed in section 5.4 and can be seen in Figure 8 .

\begin{tabular}{|l|l|}
\hline \multicolumn{2}{|c|}{ HySpex VNIR-1600 Parameters } \\
\hline IFOV across-track & $0.36 \mathrm{mrad}$ \\
\hline IFOV along-track & $0.72 \mathrm{mrad}$ \\
\hline Spectral Range & $416-992 \mathrm{~nm}$ \\
\hline $\begin{array}{l}\text { Spectral Sampling } \\
\text { and number of } \\
\text { Channels }\end{array}$ & $3.60 \mathrm{~nm}$ and 160 bands \\
\hline Spatial Pixels & 1600 \\
\hline Pixel Quantization & 12 bit \\
\hline \multicolumn{2}{|l|}{ Table 3. Parameters of the HySpex instrument } \\
\hline
\end{tabular}

\subsection{RS Correction in DESIS}

The simplest RS correction that can be applied is one that ignores any other factors and simply performs a linear interpolation between adjacent pixels in the along-track direction. The interpolation is performed for every $1024 \times 1024$ image band in the along-track direction taking into account only the phase shift $\left(\alpha^{(i)}\right)$ of that band with respect to the band chosen as reference (typically the first band acquired). This interpolation is pictured in Figure 6 and is the result of applying Equation 1.

$$
V_{j}^{R S C}{ }_{j}^{(i)}=V_{j-1}^{R S^{(i)}} \times \alpha^{(i)}+V_{j}^{R S_{j}^{(i)}} \times\left(1-\alpha^{(i)}\right)
$$

$\mathrm{V}_{\mathrm{j}}^{(\mathrm{i})}$ represents the calibrated pixel values in band $\mathrm{i}$ and position $\mathrm{j}$ in the along track direction and $0 \leq \alpha^{(\mathrm{i})} \leq 1$ represents the phase shift in band i.

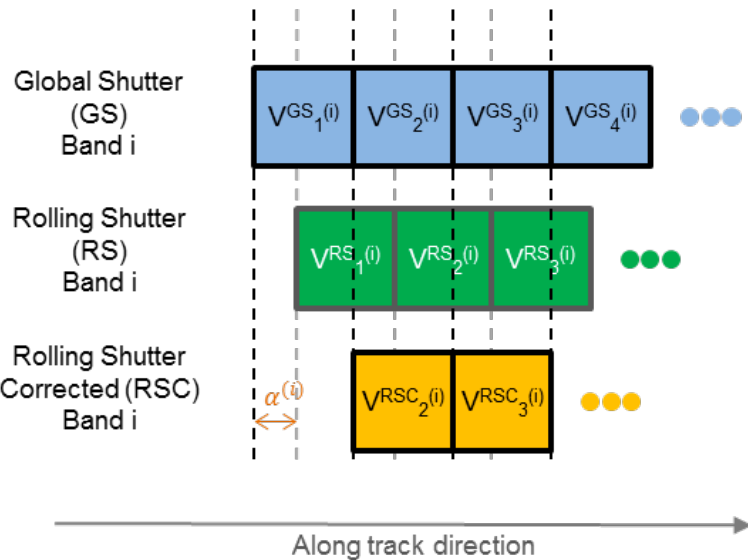

Figure 6. Illustration of RS effect in the along-track direction for band "i" with phase $\alpha^{(i)}$

In this simple model, the Correction Difference (CD) is defined as the difference between the result of the interpolation $\left(\mathrm{V}^{\mathrm{RSC}}{ }_{j}(\mathrm{i})\right.$ and the value that would have been measured in GS mode $\left(\mathrm{V}^{\mathrm{GS}(\mathrm{i})}\right)$ :

$$
C D_{j}^{(i)}=V_{j}^{G S_{j}^{(i)}}-V_{j}^{R S C}(i)
$$

This difference approaches zero for bands where $\alpha$ is near 0 or 1 and it is maximum in those bands where $\alpha$ is close to 0.5 . In case of no difference between adjacent RS pixels the RSC result shall be identical to the GS value. The maximum correction difference is expected when there is a sharp edge perpendicular to the along-track direction.
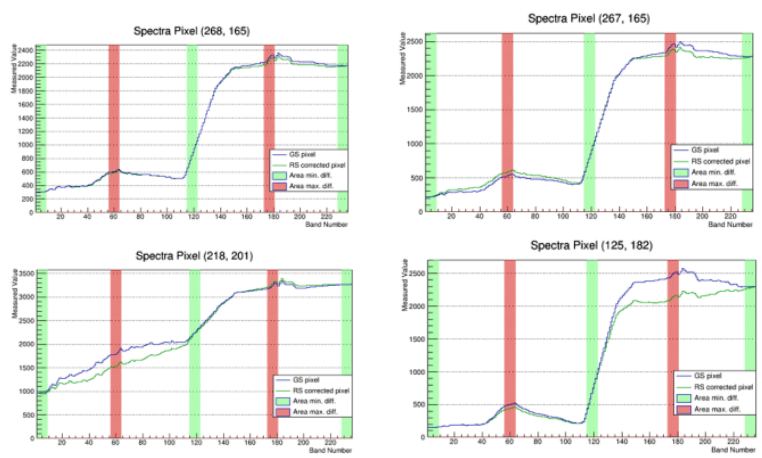

Figure 7. Simulated GS (blue line) and RSC spectra (green line) of four different $30 \mathrm{~m} \times 30 \mathrm{~m}$ DESIS pixels exhibiting different $\mathrm{CD}$ values. The pixels have been selected to show increasing GS-RSC difference levels from top-left to bottom-right. Noise is not included in this simulation

In order to illustrate what are the differences to be expected between a GS and a RSC spectra, Figure 7 shows four cases ordered from small to large $\mathrm{CD}$ values. It is worth noting that 
the discrepancies between a GS scene and a RSC scene will depend on the type of scene. Homogeneous scenes will be less affected by correction differences, while scenes with high spatial variability will be more affected, especially in those areas where one can find strong transitions between different surface materials.

At the moment this simple model represents the baseline for the RSC to be applied to DESIS data. Although it is reasonable to assume that more elaborated methods would deliver better results, we shall also consider the question of the cost of the correction in a production environment. A typical DESIS product consists of a cube of $1024 \times 1024 \times 235$ pixels (typical size $\sim 480$ Mbytes without compression) that demands considerable resources in order to be processed with complex algorithms. The baseline correction currently adopted however it is extremely simple and fast to apply.

Further investigations are in progress to find a more suitable correction for the RS effect (e.g. different interpolation methods, more elaborated techniques). For the time being, the baseline correction will be implemented as an additional step of the L1B processor as indicated in section 4.3. Results, based on simulations, obtained with this approach in the context of spectral unmixing are shown in the next section.

\subsection{Rolling Shutter Effect on Spectral Unmixing}

The following experiment has been carried out in order to evaluate the impact of spectral distortions caused by rolling shutter acquisition in practical applications. Figure 8 shows a true colour combination of an airborne HySpex image acquired over DLR premises in Oberpfaffenhofen, Germany.

From the HySpex data a $39 \times 39$ DESIS-pixels image is simulated by spectral and spatial resampling as described in section 5.2. For each DESIS pixel, an endmember extraction algorithm was run on the original HySpex image elements in the same area. We chose Vertex Component Analysis (Nascimento 2005), which needs as input parameter the number of endmembers to be extracted. We selected the highest output of the (Bioucas-Dias 2008) algorithm applied to the image patches, which was 6 .

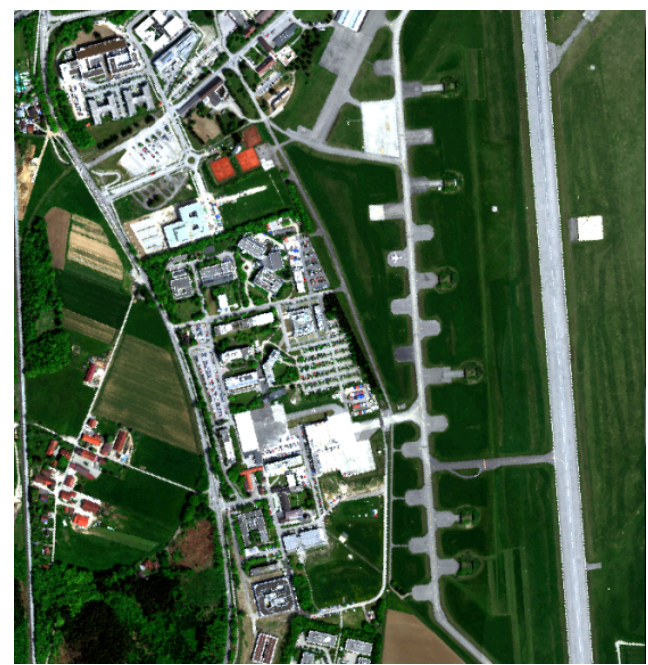

Figure 8. True colour composition of HySpex image acquired over DLR premises at Oberpfaffenhofen, Germany
Subsequently, abundances were computed on the HySpex image for each patch by inversion through Non-Negative Least Squares (NNLS) and selected as reference data. The same abundance estimation step was applied to the simulated DESIS image without rolling shutter distortions, henceforth named DESIS GS30. As distortion measure, Root Mean Squared Error (RMSE) was computed on the abundances as computed from the DESIS_GS30 image, resulting in a mean error of 0.10 .

After simulating an acquisition with RS assuming a flight direction towards North, the DESIS_RS image was simulated, and abundances were computed using the same endmember set and NNLS algorithm. The resulting RMSE was 0.23. After correcting rolling shutter effects by linear interpolation, the image DESIS_RSC was simulated, and the computed errors on the abundances were 0.17 in average.
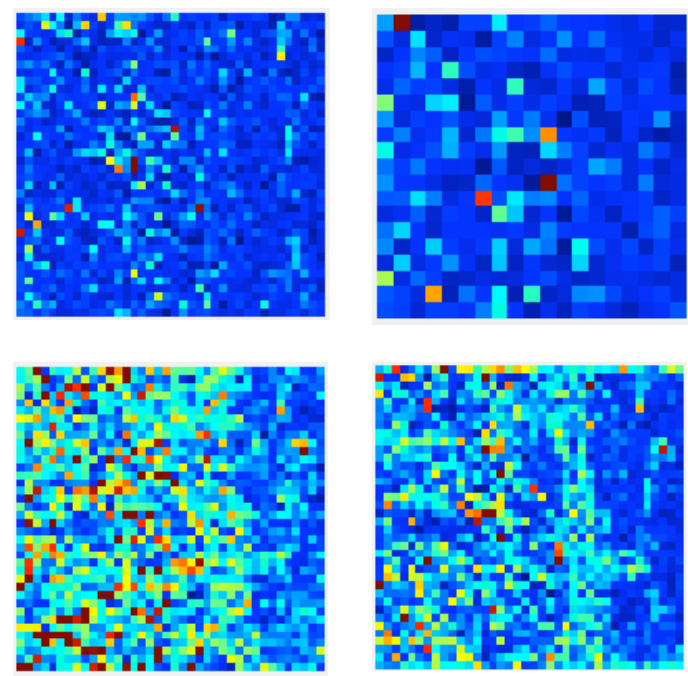

Figure 9. RMSE of the difference abundance estimations on HySpex image shown in Figure 8 for cases DESIS_GS30 (topleft), DESIS_GS60 (top right), DESIS_RS (bottom left) and DESIS_RSC (bottom right)

An additional case studied is an image acquired with GS mode at a coarser spatial resolution of $60 \mathrm{~m}$ (DESIS_GS60). This configuration would be achievable in practice by DESIS as an experimental mode. Here, the errors in abundances estimation after an endmember extraction run on the area covered by each pixel yielded mostly the same RMSE as in the case of the DESIS_GS30, suggesting that practical applications could benefit from an additional acquisition mode with these characteristics. Figure 9 shows the RMSE in each of the four cases simulated from the original HySpex image. Table 4 summarizes the results obtained in the four studied cases.

\begin{tabular}{|l|l|c|}
\hline \multicolumn{1}{|c|}{ Case } & \multicolumn{1}{c|}{ Parameters } & RMSE \\
\hline DESIS_GS30 & $30 \mathrm{~m}$ pixel, GS mode & 0.10 \\
\hline DESIS_GS60 & $60 \mathrm{~m}$ pixel, GS mode & 0.10 \\
\hline DESIS_RS & $30 \mathrm{~m}$ pixel, no RS correction & 0.23 \\
\hline DESIS_RSC & $\begin{array}{l}30 \mathrm{~m} \text { pixel, linear } \\
\text { interpolation correction } \\
\text { (baseline) }\end{array}$ & 0.17 \\
\hline
\end{tabular}

Table 4. Error (RMSE) on abundances estimation for the different simulated cases 


\subsection{RS Effect Summary}

The results obtained in our investigations suggest that the baseline correction here presented improves the results with respect to the non-RSC case. There is still margin for further improvements in order to achieve a RSC image closer to the GS results. Further investigations are required to find a more accurate and efficient RSC that can improve the results of the baseline correction.

It is however worth noting that our studies currently underestimate other factors that may be of interest in other applications and are favoured by the use of RS like the reduced noise level, high spectral resolution or the higher spatial resolution.

\section{CONCLUSION}

From 2018 onward the DESIS sensor on board the ISS will provide hyperspectral data for commercial and scientific applications. DLR is developing a processing chain in order to generate well-calibrated data products up to orthorectified and atmospheric corrected data, that include as well metadata and quality control information. Motivated by the use of a rolling shutter, this chain shall incorporate a novel procedure that it is presently being investigated and tested on simulated data.

\section{REFERENCES}

Guanter, L., et al. (2015) The EnMAP Spaceborne Imaging Spectroscopy Mission for Earth Observation. Remote Sensing (7), pp. 8830-8857. MDPI. DOI: $10.3390 /$ rs70708830. ISSN 2072-4292.

Perkins, R., et al. (2016) "Teledyne's Multi-User System for Earth Sensing (MUSES)", JACIE (Joint Agency Commercial Imagery Evaluation) Workshop, Fort Worth, Texas, USA.

Müller, R., et. al. (2016) "The New Hyperspectral Sensor DESIS on the Multi-Payload Platform MUSES Installed on the ISS", ISPRS - International Archives of the Photogrammetry, Remote Sensing and Spatial Information. Sciences, Volume XLI-B1, 2016, pp. 461-467.

USGS 2016 Landsat 8 OLI (Operational Land Imager) https://lta.cr.usgs.gov/L8 (access 5th April 2016)

Richter, R., (1996) A spatially adaptive fast atmospheric correction algorithm. International Journal of Remote Sensing, 17(6), pp. 1201-1214

Richter, R. (2011) Atmospheric Correction Methods for Optical Remote Sensing Imagery of Land. In: Advances in Environmental Remote Sensing Remote Sensing Applications. Taylor \& Francis, London. Seiten 161-172. ISBN 978-1-42009175-5.

Müller, R., Krauß, T., Schneider, M., Reinartz, P. (2012) Automated Georeferencing of Optical Satellite Data with Integrated Sensor Model Improvement. Photogrammetric Engineering and Remote Sensing (PE\&RS), 78 (1), Seiten 6174. American Society for Photogrammetry and Remote Sensing. ISSN 0099-1112.

Makarau, A., et al. (2014a) Haze detection and removal in remotely sensed multispectral imagery. IEEE Transactions on
Geoscience and Remote Sensing, 52 (9), Seiten 5895-5905. IEEE Xplore. DOI: 10.1109 /TGRS.2013.2293662. ISSN 0196-2892.

Makarau, A., et al. (2014b) Spectrally consistent haze removal in multispectral data. In: Image and Signal Processing for Remote Sensing XX, 9244, Seiten 1-7. SPIE. SPIE Remote sensing Europe, 22-25 September 2014, Amsterdam, Netherlands. ISBN 9781628413076.

J. M. P. Nascimento and J. M. B. Dias, "Vertex component analysis: a fast algorithm to unmix hyperspectral data," IEEE Transactions on Geoscience and Remote Sensing, vol. 43, no. 4, pp. 898-910, 2005.

J. Bioucas-Dias, J. Nascimento, "Hyperspectral Subspace Identification", IEEE Transactions on Geoscience and Remote Sensing, vol.46, no.8, pp.2435-2445, 2008. 